\title{
Test-Retest Reliability of Frontal and Parietal Alpha Asymmetry during Presentation of Emotional Face Stimuli in Healthy Subjects
}

\author{
Kristin Koller-Schlaud ${ }^{\mathrm{a}, \mathrm{b}} \quad$ Julia Querbach ${ }^{\mathrm{a}} \quad$ Joachim Behr ${ }^{\mathrm{a}-\mathrm{c}}$ Andreas Ströhle ${ }^{\mathrm{a}}$ \\ Johannes Rentzsch ${ }^{a, b}$ \\ ${ }^{a}$ Department of Psychiatry and Psychotherapy Campus Mitte, Charité - Universitätsmedizin Berlin, corporate \\ member of Freie Universität Berlin, Humboldt-Universität zu Berlin, and Berlin Institute of Health, Berlin, Germany; \\ ${ }^{b}$ Department of Psychiatry, Psychotherapy, and Psychosomatics, Brandenburg Medical School Theodor Fontane, \\ Neuruppin, Germany; ' Faculty of Health Science Brandenburg, Joint Faculty of the University of Potsdam, \\ Brandenburg University of Technology Cottbus-Senftenberg and Brandenburg Medical School Theodor Fontane, \\ Potsdam, Germany
}

\section{Keywords}

Reliability · EEG · Frontal alpha asymmetry · Parietal alpha asymmetry $\cdot$ Alpha asymmetry

\section{Abstract \\ Resting-state and event-related frontal alpha asymmetry have been suggested as potential neurobiological biomark- ers for depression and other psychiatric conditions. To be used as such, sufficient test-retest reliability needs to be demonstrated. However, test-retest reliability is underinves- tigated for event-related alpha asymmetry. The objective of this study was to examine both short-term within-session and long-term between-session reliability of stimulus-relat- ed medial and lateral frontal as well as parietal alpha EEG asymmetry in healthy subjects during a simple emotional face processing task. Twenty-three healthy adults participat- ed in two sessions with a test-retest interval of about 1 week. Reliability was estimated with Pearson's correlation coeffi- cient and paired $t$ test. Results revealed moderate to high within-session reliability of stimulus-related alpha asymme- try for all electrode sites and both conditions. Alpha asym- metry mean values did not change significantly within ses-}

(C) 2020 S. Karger AG, Basel

www.karger.com/nps

Karger ${ }^{\prime}=$ sions. Between-session reliability was fair for frontomedial and moderate for frontolateral stimulus-related asymmetry. Exploratory exclusion of subjects with unstable betweensession self-rating scores of emotional state and empathy toward stimuli resulted in some higher reliability values. Our results indicate that stimulus-related alpha asymmetry may serve as a useful electrophysiological tool given its adequate within-session reliability. However, long-term stability of stimulus-related frontal alpha asymmetry over 1 week was comparatively low and varied depending on electrode position. Influencing state factors during EEG recording, such as current mood or stimulus engagement, should be considered in future study designs and analyses. Further, we recommend to analyze alpha asymmetry from both frontomedial and frontolateral sites.

(c) 2020 S. Karger AG, Basel

\section{Introduction}

Impairments in emotional processing have been associated with several psychiatric disorders [1-4]. Studies have for instance found decreased accuracy in recognition 
of stimulus valence in unipolar depression [5-7], bipolar disorders $[8,9]$, and schizophrenia $[3,4]$. There is evidence that frontal cortex areas, among other core domains of the emotion-processing network, may be associated with emotion recognition and processing bias $[3,10]$.

Resting frontal alpha asymmetry - the hemispheric difference in the right versus the left power of the alpha frequency band at frontal electrodes under resting condition - has been investigated as a potential neurobiological biomarker for psychiatric disorders, e.g., affective [11$15]$ and anxiety disorders [16,17], attention deficit hyperactivity disorder [18], and schizophrenia [13]. Several studies have found evidence that individuals with major depressive episodes show more relative left than right hemispheric frontal alpha power compared to healthy controls [19-22]. However, recent studies have reported limited diagnostic value of resting alpha asymmetry in unipolar depression, potentially due to differences in methodology and clinical characteristics of investigated samples across studies [17, 23]. It has been suggested that resting frontal alpha asymmetry may be more useful in investigating specific symptom clusters of psychiatric disorders than diagnostic entities $[16,23]$. Others have suggested that measuring alpha asymmetry under emotional task manipulation rather than under resting condition might produce more consistent results by reducing uncontrolled variance [24].

In healthy individuals it was found that frontal alpha asymmetry was associated with emotional processing and related to motivational direction [25]. Stress induction was associated with more relative left than right hemispheric frontal alpha power [26]. Interestingly, healthy individuals who showed this asymmetric pattern of frontal alpha power rated emotional stimuli as more negative [27, 28]. On the other hand, stimuli which are thought to be approach-related are associated with relatively greater right than left alpha power in healthy individuals [29-32]. Overall, these studies indicate that resting as well as stimulus-related frontal alpha asymmetry is an important neurophysiological parameter in the investigation of emotion and behavior in healthy and clinical populations [16].

One important precondition for the use of frontal alpha asymmetry as a neurobiological marker is sufficient long-term stability. This is also of importance to allow for correct interpretations of changes in alpha asymmetry during the course of an illness or during early treatment response in, e.g., depressive episodes [19]. Test-retest studies in healthy individuals have shown between-session correlation of alpha asymmetry scores of about $0.4-$ 0.7 for the midfrontal electrodes for up to 6 weeks $[22,28$,
33,34 ]. Hagemann et al. [35] found that about $60 \%$ of the variance in resting frontal alpha asymmetry was temporally stable and that alpha asymmetry reflects both trait and state characteristics. Anokhin et al. [36] found that about $27 \%$ of variance in midfrontal alpha asymmetry can be accounted for by genetic factors. Interestingly, Wheeler et al. [37] found that emotional reactivity evoked by short film clips after EEG recording was related to alpha asymmetry only in individuals with stable test-retest alpha asymmetry.

Reliability is crucial if stimulus-related alpha asymmetry is to be used as a neurobiological marker. However, while reliability of resting condition alpha asymmetry was investigated in numerous studies, no study has yet to our best knowledge published short- and long-term testretest reliability of stimulus-related alpha asymmetry.

The aim of this study was to examine both short-term within-session and long-term between-session reliability of frontal alpha asymmetry in a simple emotional face processing paradigm with alternating trains of happy and sad face stimuli in healthy subjects. Alternating emotional stimuli were used to investigate whether transient changes in emotional valence had an impact on reliability of alpha asymmetry, which is increasingly used as a neurobiological index for emotional processing and behavioral approach motivation [25] and as a marker for psychiatric disorders. Face stimuli are commonly used in studies to investigate emotion-related neuronal activity. For example, studies on affective disorders have shown that depressed individuals show selective attention toward sad expressions and reduced attention for happy facial expressions [1]. In the current study, we investigated nondepressed healthy individuals as these often serve as a reference control group, and thus we did not assume a response bias to either positive or negative face stimuli. Alpha asymmetry was measured both at medial and lateral frontal electrodes, as there is evidence that happy and sad face stimuli processing is differentially associated with frontomedial and frontolateral brain activity [38]. To estimate whether reliability was specific to particular frontal sites or rather an estimate of a general reliability, we also assessed asymmetry on parietal electrode sites.

\section{Subjects and Methods}

\section{Subjects}

A total of 23 healthy adults (12 female, 11 male; mean age \pm standard deviation $27 \pm 10.8$ years) participated in the study. All subjects had completed secondary-level education. Exclusion criteria were axis I psychiatric diagnoses according to DSM-IV (Mini- 
International Neuropsychiatric Interview [39]), except for nicotine and caffeine dependence. Further exclusion criteria were severe internal or neurological diseases, known but untreated hypothyroidism or diabetes mellitus, individuals using any psychotropic medication, and individuals using more than two nonpsychotropic prescription medications a day. Individuals with any known axis II psychiatric disorders were also excluded; however, this was not assessed using a structural clinical psychiatric interview.

\section{Procedure}

Subjects participated in two experimental sessions separated by approximately 7 days $(6.7 \pm 2.2$; difference of time of day between sessions $1.9 \pm 2.1)$. Participants were seated in a comfortable chair with a headrest in a room adjacent to the recording apparatus. The experimental task lasted about 13 min including breaks. Before the first session, criteria for axis I psychiatric diagnosis were checked, demographic information was obtained, and the participants completed the Beck Depression Inventory version 2 (BDI-II) to assess subclinical depressive symptoms (BDI score $2.0 \pm 2.3$, range $0-6$ ).

\section{Task}

The task consisted of alternating trials showing a consecutive series of either sad or happy gender-randomized facial expressions (online suppl. Fig. 1; for all online suppl. material, see www. karger.com/doi/10.1159/000505783). One trial consisted of a series of five to eight faces of one emotion. To control for attention, participants were instructed to press a button as they noticed a switch from one emotional expression to the other, i.e., a switch from the happy to the sad or from the sad to the happy trial. After six trials the participants were asked, "In how far were you able to empathize with the faces?" followed by a short break of about $10 \mathrm{~s}$ until the next six trials were presented. Subjects self-reported to what extent they felt empathy toward the face stimuli on a Likert scale ranging from 1 (very much) to 4 (very little).

After 18 trials the participants were also asked, "How do you feel?" followed by a long break of about $20 \mathrm{~s}$. Subjects self-reported how they themselves felt on a Likert scale ranging from 1 (very good) to 4 (very bad). Overall, there were 36 trials of sad and 36 trials of happy facial expressions and 8 short and 3 long breaks (235 \pm 6.5 face stimuli per emotion; duration of face stimuli presentation: 1,000 ms). We used happy and sad emotional face stimuli from the Karolinska Directed Emotional Faces database which have been evaluated on emotional content, intensity, and arousal [40].

\section{Data Collection and Analysis}

Continuous EEG was recorded during visual stimulus presentation from 29 scalp locations using an electrode cap following the International 10/20 system with additional electrodes (BrainAmp recorder, Brain Products GmbH, Gilching, Germany; EasyCap, EASYCAP GmbH, Herrsching, Germany). Fpz served as ground and $\mathrm{Afz}$ as reference. Electrode impedance was $<10 \mathrm{k} \Omega$. Data were collected at a sampling rate of $500 \mathrm{~Hz}$ (gain 5,000; analog bandpass filter $0.15-100 \mathrm{~Hz}$ ).

Offline EEG analysis was performed using Brain Vision Analyzer version 2.1 (Brain Products $\mathrm{GmbH}$ ) and EEGLAB version 13.1.1 [41]. Following offline filtering of the continuous EEG signal (Butterworth band-pass $0.5-30 \mathrm{~Hz}$, noise filter $50 \mathrm{~Hz}, 12 \mathrm{~dB}$ ), transient artifacts (e.g., ocular or other muscular movements and electrocardiac artifacts) were automatically corrected using the EEGLAB plugins Artifact Subspace Reconstruction version 0.13 [42] and Automatic Artifact Removal version 1.3 [43]. In some instances, eye movements and electrocardiac artifacts could not be corrected using automatic procedures. In these cases, ICA-based correction was performed by manual elimination of the independent components corresponding to eye movements and electrocardiac artifacts. After transforming to average reference and artifact rejection $( \pm 100 \mu \mathrm{V})$, the continuous EEG signal was segmented (1,000 ms after stimulus onset) and fast Fourier transformed (Hamming window, windows length $10 \%$ with variance correction, periodic windows function; resolution $0.49 \mathrm{~Hz}$ ). The FFT signal for each segment was averaged without overlap, separated for the first and second half of each test session. These were also averaged separately for the happy and sad face conditions. Power for the alpha frequency band $(8-13 \mathrm{~Hz})$ was extracted for left and right frontal and parietal electrodes (frontomedial, F3/F4; frontolateral, F7/F8; parietomedial, P3/P4; parietolateral, P7/P8) for further statistical analysis.

Alpha asymmetry scores were computed by subtracting left from right ln-transformed mean power values. Processing and analysis were performed in a blinded fashion regarding time of session.

There was no significant difference between segments after artefact rejection, either within or between sessions (within sessions, first versus second block of both sessions: happy face condition, $224 \pm 5$ vs. $226 \pm 5, t=-1.6, p>0.1$; sad face condition, $223 \pm 6$ vs. $225 \pm 5, t=-1.2, p>0.1$; between sessions, first versus second session: happy face condition, $224 \pm 5$ vs. $226 \pm 6, t=-1.3, p>0.1$; sad face condition, $224 \pm 4$ vs. $223 \pm 6, t=-0.5, p>0.1$ ).

\section{Data Analyses and Statistics}

All statistical analyses were conducted using the Statistical Package for Social Sciences (SPSS version 15.0 for Windows; SPSS Inc., Chicago, IL, USA). All parameters are presented as mean and standard deviations as appropriate. Test-retest reliability was estimated with Pearson's correlation coefficient within sessions (first versus second halves of both sessions) and between sessions (entire first session versus entire second session) separately for the two emotional face conditions (happy and sad faces). Reliability was defined as follows: correlation coefficient ranges $0.0-0.10$, virtually none; $0.11-0.40$, slight; $0.41-0.60$, fair; $0.61-0.80$, moderate; 0.81-1.0 high [44]. Intraclass correlation coefficients with consistency over all test sessions within conditions were reported for descriptive purposes. Statistical significance was defined as $p<$ 0.05. All tests were two-tailed. Comparison of within- and between-session power values was conducted using $t$ tests without correcting for multiple testing. We did this on the basis that correction for multiple testing would introduce a bias toward a conclusion of no differences between and within sessions.

\section{Results}

\section{Self-Ratings and Behavioral Data}

Self-Ratings. Data and statistical analyses of self-ratings are shown in Table 1. Self-rated emotional state decreased slightly within but not between sessions. Self-rat- 
Table 1. Self-ratings (mean \pm SD) and statistical comparisons

\begin{tabular}{|c|c|c|c|c|c|c|c|c|c|}
\hline & \multicolumn{4}{|c|}{ Within sessions } & \multicolumn{4}{|c|}{ Between sessions } & \multirow{2}{*}{$\begin{array}{l}\text { All sessions, } \\
\text { ICC (95\% CI) }\end{array}$} \\
\hline & block 1 & block 2 & Pearson & $t$ test & session 1 & session 2 & Pearson & $t$ test & \\
\hline $\begin{array}{l}\text { Emotional state } \\
\text { Empathy toward faces }\end{array}$ & $1.72 \pm 0.55$ & $1.79 \pm 0.54$ & $0.97^{* * * *}$ & $-2.4^{* *}$ & $1.74 \pm 0.52$ & $1.77 \pm 0.63$ & $0.78^{* * * *}$ & $-0.32^{\mathrm{ns}}$ & $0.79(0.65-0.89)$ \\
\hline Нарру & $1.67 \pm 0.51$ & $1.83 \pm 0.56$ & $0.89^{* * * *}$ & $-2.8^{* *}$ & $1.67 \pm 0.54$ & $1.83 \pm 0.58$ & $0.73^{* * * *}$ & $-1.78^{*}$ & $0.70(0.53-0.84)$ \\
\hline Sad & $1.99 \pm 0.66$ & $2.11 \pm 0.63$ & $0.90^{* * * *}$ & $-1.9 *$ & $2.09 \pm 0.70$ & $2.01 \pm 0.62$ & $0.81^{* * * *}$ & $0.88^{\mathrm{ns}}$ & $0.78(0.63-0.88)$ \\
\hline Overall & $1.83 \pm 0.54$ & $1.97 \pm 0.56$ & $0.89^{* * * *}$ & $-2.6^{* *}$ & $1.88 \pm 0.56$ & $1.92 \pm 0.57$ & $0.69^{* * * *}$ & $-0.39^{n s}$ & $0.65(0.50-0.80)$ \\
\hline
\end{tabular}

Within- and between-session (Pearson's $R$ ) and overall reliability (ICC with 95\% CI, model 3.1 with consistency). ICC, intraclass correlation coefficient. ${ }^{*} p<0.1,{ }^{* *} p<0.05$, ${ }^{* * * *} p<0.001,{ }^{\text {ns }} p \geq 0.1$. ${ }^{1}$ Emotional state ("How do you feel?"): higher values indicate lower ratings of self-rated emotional state. ${ }^{2}$ Empathy toward faces ("In how far were you able to empathize with the faces?"): higher values indicate lower ratings of empathy toward the face stimuli.

Table 2. Alpha power $\left(\mu \mathrm{V}^{2}\right)$ for frontomedial $(\mathrm{F} 3, \mathrm{~F} 4)$ and frontolateral $(\mathrm{F} 7, \mathrm{~F} 8)$ electrodes (mean $\left.\pm \mathrm{SD}\right)$ and statistical comparisons

\begin{tabular}{|c|c|c|c|c|c|c|c|c|c|}
\hline & \multicolumn{4}{|c|}{ Within sessions } & \multicolumn{4}{|c|}{ Between sessions } & \multirow{2}{*}{$\begin{array}{l}\text { All sessions, } \\
\text { ICC }(95 \%)\end{array}$} \\
\hline & block 1 & block 2 & Pearson & $t$ test & session 1 & session 2 & Pearson & $t$ test & \\
\hline \multicolumn{10}{|c|}{ Left frontomedial } \\
\hline Happy & $0.285 \pm 0.172$ & $0.317 \pm 0.209$ & $0.98^{* * * *}$ & $-2.72 * *$ & $0.282 \pm 0.174$ & $0.321 \pm 0.227$ & $0.79 * * * *$ & $-1.34^{\mathrm{ns}}$ & $0.87(0.77-0.94)$ \\
\hline Sad & $0.292 \pm 0.190$ & $0.308 \pm 0.199$ & $0.97^{* * * *}$ & $-1.51^{\mathrm{ns}}$ & $0.278 \pm 0.175$ & $0.322 \pm 0.226$ & $0.85^{* * * *}$ & $-1.76^{*}$ & $0.89(0.81-0.95)$ \\
\hline \multicolumn{10}{|c|}{ Right frontomedial } \\
\hline Happy & $0.299 \pm 0.188$ & $0.329 \pm 0.201$ & $0.99^{* * * *}$ & $-3.88^{* * * *}$ & $0.288 \pm 0.171$ & $0.341 \pm 0.228$ & $0.88^{* * * *}$ & $-2.29 * *$ & $0.93(0.87-0.96)$ \\
\hline Sad & $0.309 \pm 0.202$ & $0.327 \pm 0.206$ & $0.97^{* * * *}$ & $-1.67^{\mathrm{ns}}$ & $0.297 \pm 0.193$ & $0.339 \pm 0.221$ & $0.91^{* * * *}$ & $-2.16^{* *}$ & $0.92(0.86-0.96)$ \\
\hline \multicolumn{10}{|c|}{ Left frontolateral } \\
\hline Нарру & $0.426 \pm 0.429$ & $0.473 \pm 0.499$ & $0.99^{* * * *}$ & $-2.73^{* *}$ & $0.395 \pm 0.339$ & $0.505 \pm 0.611$ & $0.89^{* * * *}$ & $-1.52^{\mathrm{ns}}$ & $0.92(0.85-0.96)$ \\
\hline Sad & $0.418 \pm 0.411$ & $0.461 \pm 0.491$ & $0.99^{* * * *}$ & $-1.98^{*}$ & $0.375 \pm 0.309$ & $0.504 \pm 0.634$ & $0.79 * * * *$ & $-1.44^{\mathrm{ns}}$ & $0.91(0.84-0.99)$ \\
\hline \multicolumn{10}{|c|}{ Right frontolateral } \\
\hline Happy & $0.357 \pm 0.234$ & $0.385 \pm 0.244$ & $0.98^{* * * *}$ & $-2.73^{* *}$ & $0.323 \pm 0.157$ & $0.419 \pm 0.348$ & $0.73^{* * * *}$ & $-1.79 *$ & $0.84(0.73-0.92)$ \\
\hline Sad & $0.355 \pm 0.215$ & $0.372 \pm 0.233$ & $0.98^{* * * *}$ & $-1.67^{\mathrm{ns}}$ & $0.326 \pm 0.168$ & $0.401 \pm 0.305$ & $0.74^{* * * *}$ & $-1.71^{\mathrm{ns}}$ & $0.87(0.77-0.93)$ \\
\hline
\end{tabular}

Within- and between-session (Pearson's $R$ ) and overall reliability (ICC with 95\% CI, model 3.1 with consistency). ICC, intraclass correlation coefficient. ${ }^{*} p<0.1,{ }^{* *} p<0.05,{ }^{* * * *} p<0.001,{ }^{\mathrm{ns}} p \geq 0.1$.

ed empathy toward faces decreased for the happy condition with a statistical significance within sessions and with a statistical trend between sessions. There was a statistical trend for reduced empathy toward sad faces within sessions and no statistically significant difference between sessions.

Behavioral Data. Data and statistical analyses of behavioral data are shown in supplementary Tables 1 and 2 . There were no significant differences between the number of correctly identified changes in faces' emotion within and between sessions. Reaction time tended to be slower for the second compared to the first block of sessions for both face conditions. Correlations between behavioral data and alpha power and asymmetry values are shown for descriptive purposes in supplementary Tables 3 and 4. Reaction time of the sad but not of the happy face condition was positively correlated with alpha power at most electrodes for the second but not first block and session. Alpha asymmetry was negatively correlated with reaction time in the sad face condition for the frontolateral sites for block 2 and session 2 .

\section{Electrophysiological Results}

The alpha power values and the within- and betweensession comparisons are shown for frontal and parietal alpha power and alpha asymmetry in Tables $2-4$, respectively. Online supplementary Figure 2 shows topographic maps of alpha power for each block and session. 
Table 3. Alpha power $\left(\mu \mathrm{V}^{2}\right)$ for parietomedial $(\mathrm{P} 3, \mathrm{P} 4)$ and parietolateral (P7, P8) electrodes (mean \pm SD) and statistical comparisons

\begin{tabular}{|c|c|c|c|c|c|c|c|c|c|}
\hline & \multicolumn{4}{|c|}{ Within sessions } & \multicolumn{4}{|c|}{ Between sessions } & \multirow{2}{*}{$\begin{array}{l}\text { All sessions, } \\
\text { ICC (95\% CI) }\end{array}$} \\
\hline & block 1 & block 2 & Pearson & $t$ test & session 1 & session 2 & Pearson & $t$ test & \\
\hline \multicolumn{10}{|c|}{ Left parietomedial } \\
\hline Happy & $0.424 \pm 0.278$ & $0.472 \pm 0.315$ & $0.98^{* * * *}$ & $-3.04^{* * *}$ & $0.373 \pm 0.219$ & $0.524 \pm 0.399$ & $0.79^{* * * *}$ & $-2.79 * *$ & $0.92(0.86-0.98)$ \\
\hline Sad & $0.445 \pm 0.318$ & $0.475 \pm 0.331$ & $0.93^{* * * *}$ & $-1.15^{\mathrm{ns}}$ & $0.392 \pm 0.255$ & $0.528 \pm 0.421$ & $0.76^{* * * *}$ & $-2.31^{* *}$ & $0.88(0.78-0.94)$ \\
\hline \multicolumn{10}{|c|}{ Right parietomedial } \\
\hline Happy & $0.402 \pm 0.233$ & $0.462 \pm 0.280$ & $0.97^{* * * *}$ & $-3.54^{* * *}$ & $0.368 \pm 0.202$ & $0.496 \pm 0.334$ & $0.78^{* * * *}$ & $-2.82^{* *}$ & $0.87(0.77-0.94)$ \\
\hline Sad & $0.411 \pm 0.249$ & $0.449 \pm 0.272$ & $0.95^{* * * *}$ & $-2.09 * *$ & $0.363 \pm 0.207$ & $0.497 \pm 0.833$ & $0.79^{* * * *}$ & $-3.01^{* * *}$ & $0.86(0.76-0.93)$ \\
\hline \multicolumn{10}{|c|}{ Left parietolateral } \\
\hline Happy & $0.536 \pm 0.355$ & $0.584 \pm 0.406$ & $0.98^{* * * *}$ & $-2.35^{* *}$ & $0.461 \pm 0.241$ & $0.660 \pm 0.556$ & $0.76^{* * * *}$ & $-2.36^{* *}$ & $0.83(0.71-0.91)$ \\
\hline Sad & $0.542 \pm 0.353$ & $0.575 \pm 0.417$ & $0.96^{* * * *}$ & $-1.29^{\mathrm{ns}}$ & $0.461 \pm 0.249$ & $0.655 \pm 0.562$ & $0.74^{* * * *}$ & $-2.23^{* *}$ & $0.83(0.71-0.92)$ \\
\hline \multicolumn{10}{|c|}{ Right parietolateral } \\
\hline Happy & $0.543 \pm 0.320$ & $0.611 \pm 0.380$ & $0.97 * * * *$ & $-3.22^{* * *}$ & $0.502 \pm 0.282$ & $0.653 \pm 0.449$ & $0.79^{* * * *}$ & $-2.58^{* *}$ & $0.87(0.77-0.94)$ \\
\hline Sad & $0.564 \pm 0.346$ & $0.606 \pm 0.387$ & $0.94^{* * * *}$ & $-1.46^{\mathrm{ns}}$ & $0.506 \pm 0.299$ & $0.665 \pm 0.457$ & $0.81^{* * * *}$ & $-2.74^{* *}$ & $0.86(0.76-0.93)$ \\
\hline
\end{tabular}

Within- and between-session (Pearson's $R$ ) and overall reliability (ICC with 95\% CI, model 3.1 with consistency). ICC, intraclass correlation coefficient. ${ }^{* *} p<0.05,{ }^{* * *} p<0.01,{ }^{* * * *} p<0.001,{ }^{n s} p \geq 0.1$.

Table 4. Alpha asymmetry values for medial and lateral, frontal and parietal brain sites (mean \pm SD) and statistical comparisons

\begin{tabular}{|c|c|c|c|c|c|c|c|c|c|}
\hline & \multicolumn{4}{|c|}{ Within sessions } & \multicolumn{4}{|c|}{ Between sessions } & $\begin{array}{l}\text { All sessions, } \\
\text { ICC }(95 \%)\end{array}$ \\
\hline Happy & $0.029 \pm 0.177$ & $0.052 \pm 0.161$ & $0.77^{* * * *}$ & $-0.93^{\mathrm{ns}}$ & $0.019 \pm 0.210$ & $0.070 \pm 0.149$ & $0.42^{* *}$ & $-1.23^{\mathrm{ns}}$ & $0.50(0.29-0.71)$ \\
\hline Sad & $0.045 \pm 0.161$ & $0.058 \pm 0.129$ & $0.89^{* * * *}$ & $-0.84^{\mathrm{ns}}$ & $0.046 \pm 0.187$ & $0.062 \pm 0.126$ & $0.45^{* *}$ & $-0.45^{\mathrm{ns}}$ & $0.53(0.33-0.73)$ \\
\hline \multicolumn{10}{|c|}{ Frontolateral } \\
\hline \multicolumn{10}{|c|}{ Parietomedial } \\
\hline Happy & $-0.007 \pm 0.229$ & $0.014 \pm 0.247$ & $0.86^{* * * *}$ & $-0.78^{\mathrm{ns}}$ & $0.008 \pm 0.242$ & $0.002 \pm 0.255$ & $0.75^{* * * *}$ & $0.18^{\mathrm{ns}}$ & $0.72(0.55-0.85)$ \\
\hline Sad & $-0.017 \pm 0.241$ & $-0.008 \pm 0.235$ & $0.89^{* * * *}$ & $-0.41^{\mathrm{ns}}$ & $-0.029 \pm 0.268$ & $0.001 \pm 0.232$ & $0.73^{* * * *}$ & $-0.76^{\mathrm{ns}}$ & $0.70(0.53-0.84)$ \\
\hline \multicolumn{10}{|c|}{ Parietolateral } \\
\hline Happy & $0.049 \pm 0.225$ & $0.070 \pm 0.221$ & $0.80^{* * * *}$ & $-0.73^{\text {ns }}$ & $0.065 \pm 0.267$ & $0.064 \pm 0.257$ & $0.30^{\mathrm{ns}}$ & $0.00^{\mathrm{ns}}$ & $0.44(0.23-0.66)$ \\
\hline
\end{tabular}

Within- and between-session (Pearson's $R$ ) and overall reliability (ICC with 95\% CI, model 3.1 with consistency). Asymmetry was computed as $\ln$ (right alpha power) - $\ln \left(\right.$ left alpha power). ICC, intraclass correlation coefficient. ${ }^{* *} p<0.05,{ }^{* * *} p<0.01,{ }^{* * * *} p<0.001$, ${ }^{\mathrm{n} s} p \geq 0.1$.

Frontal Alpha Power. Within-session retest reliabilities of alpha power for frontomedial and frontolateral electrodes were high for both happy and sad face stimuli (correlation coefficients: 0.97-0.99). Between-session retest reliabilities were moderate to high for both frontal electrode sites under both stimulus conditions (correlation coefficients: $0.73-0.91)$. Within sessions, alpha power values increased significantly for all alpha power values in the happy face condition, but not significantly in the sad face condition, despite a trend in this direction across both the frontomedial and frontolateral sites. Between sessions, in both conditions only the right frontomedial alpha power increased significantly over time between the first and second session. For some of the other electrode sites, an increase with a statistical trend was observed (Table 2).

Parietal Alpha Power. Within-session reliabilities were significantly high for all parietal alpha power values (reli- 
ability range: 0.93-0.98). Between-session reliabilities were significantly moderate to high (correlation coefficients: 0.74-0.81). As seen for the frontal electrode sites, parietal alpha power increased significantly within sessions for all happy-related alpha power values. Additionally, a significant increase was also observed for the sadrelated right parietomedial electrode sites. For the other sad-related conditions, however, no statistically significant changes were observed. Between sessions there was a statistically significant increase in alpha power for all parietal electrodes under all conditions (Table 3).

Frontal and Parietal Alpha Asymmetry. Within-session reliability values for frontal and parietal alpha asymmetry were high for all (correlation coefficients: 0.86$0.95)$ except the happy-related asymmetry at the frontomedial $(r=0.77)$ and parietolateral $(r=0.80)$ sites, which were moderate (Table 4). Between-session reliability was moderate for frontolateral and parietomedial asymmetry for both conditions (correlation coefficients: 0.63-0.75). Reliability was fair for all other sites and conditions (correlation coefficients: $0.41-0.45$ ) but for the happy-related parietolateral asymmetry (correlation coefficients: not significant). Asymmetry values did not differ significantly within and between sessions (Table 4).

In an exploratory partial correlation, we included age and BDI scores as control variables. However, there were no relevant changes of any asymmetry correlation coefficients (data not shown). Exclusion of subjects $(n=4)$ whose handedness score was $<60$, indicating more ambidexterity or left-handedness, did not change the correlation coefficients substantially (data not shown). We further tested whether changes in self-reports of emotional state ("How did you feel?") and of empathy with the presented face stimuli ("In how far were you able to empathize with the faces?") within and between sessions influenced reliability. This partial correlation analysis did not change correlation coefficients (data not shown). In a data-driven exploratory analysis, we excluded subjects whose self-rating scores of emotional state and empathy toward the faces differed more than 1.7 standard deviations between sessions $(n=9)$. This resulted in some higher between-session correlation coefficients for frontomedial (happy condition: $r=0.65, p<0.013$; sad condition: $r=0.61, p<0.022$ ) and frontolateral (happy condition: $r=0.87, p<0.0001$; sad condition: $r=0.77, p<0.002$ ) asymmetry. The correlation coefficients for parietal asymmetry scores did not change (parietomedial: happy condition, $r=0.79, p<0.001$; sad condition, $r=0.75, p<$ 0.001 ) or were lower than before exclusion (parietolateral: happy condition, $r=0.25, p>0.38$; sad condition: $r=$
$0.40, p>0.15)$. Subjects who were excluded from this analysis and those who were not did not differ significantly in age $\left(\mathrm{T}_{(21)}=0.12, p>0.8\right)$, handedness $\left(\mathrm{T}_{(21)}=\right.$ $1.02, p>0.32)$, ratio of female gender $\left(\chi^{2}=0.68, p>0.79\right)$, days between test sessions $\left(\mathrm{T}_{(21)}=1.2, p>0.22\right)$, or daytime of test sessions $\left(\mathrm{T}_{(21)}=1.3, p>0.19\right)$. Within-session asymmetry correlation coefficients for this sample were between 0.75 and 0.96 (all $p<0.003$ ).

\section{Discussion}

Resting and stimulus-related frontal alpha asymmetry has been increasingly used as a neurobiological index for emotional processing and behavioral approach motivation both in general and psychiatric populations $[16,25$, 45]. Reliability is a crucial requirement in the use of neurobiological markers. While resting-state alpha asymmetry reliability has been investigated in numerous studies, short- and long-term reliability of stimulus-related alpha asymmetry has not been investigated sufficiently. For that purpose, we measured stimulus-related frontal alpha asymmetry at medial and lateral frontal sites, and for comparison reasons also at parietal electrodes.

Overall, the reliability of stimulus-related alpha asymmetry, which was moderate for within-session shortterm reliability and fair to moderate for long-term between-session reliability, was in line with the results of studies investigating resting alpha asymmetry. Reliability of alpha asymmetry was observed independent of stimulus condition, i.e., presentation of happy versus sad faces. However, we revealed a varying long-term stability depending on brain region (i.e., frontal, parietal; medial, lateral). Moderate reliability of alpha asymmetry was found for the frontolateral and the parietomedial electrode sites. The low reliability of the frontomedial asymmetry may indicate that internal and/or external state factors in some subjects may affect the temporal stability in these areas. In line with this interpretation, we found better reliability values after exclusion of subjects who showed more variance in self-ratings of mood state and empathy over time in our exploratory analysis.

Other studies have reported better reliability at the frontomedial sites (moderate levels between 0.53 and $0.69)[22,28,34,46]$. On the other hand, we found better reliability for the lateral frontal asymmetry than two previous studies $[22,28]$. Low reliability could be one of the reasons for the heterogeneity of the results of studies investigating resting alpha asymmetry in depressed patients and healthy controls [23]. In accordance with Debener et 
al. [22] and Sutton and Davidson [28], parietal asymmetry reliabilities were moderate for medial but less than moderate for lateral parietal sites.

Differences in reported reliability may be related to differences between laboratories and methods applied. In a recent study, Tenke et al. [33] investigated the test-retest reliability (mean 7.6 days) of a four-center study and found mean correlations of alpha asymmetry between 0.37 and 0.70 . Notably, they also reported great differences in reliability values across study sites: for example, reliability at the frontomedial electrode position ranged between -0.09 and 0.65 (mean 0.37 ) and at the frontolateral position between 0.19 and 0.79 (mean 0.59 ). However, this may also be related to the small sample size at each study site. Despite methodological differences between our and Tenke et al.'s study (which used CSD reference and PCA techniques), they yielded a similarly high reliability of alpha power (mean range across electrode positions $0.73-$ 0.88 , range across study sites and electrode $0.32-0.98$ ).

The fact that long-term reliability differed between medial and lateral frontal electrode sites in our study may be of relevance, as some studies have collapsed resting alpha power across medial and lateral electrode sites [22, $30,47,48]$. In contrast, others have found differences or an opposite pattern in alpha asymmetry between medial and lateral frontal sites [12, 49-52]. Anokhin et al. [36] found heritability for frontomedial but not for frontolateral resting alpha asymmetry. These studies together with our results of varying reliability may indicate that medial and lateral frontal alpha asymmetry depend on different underlying processes.

The limited long-term reliability for some asymmetry scores was not caused by low alpha power stability in our study, as alpha power showed moderate to high longterm reliability across all conditions and electrode sites. The alpha power long-term reliability in the present study was in the range of the reliability of evoked potential across different paradigms (range: 0.74-0.91) [53] and was quite similar to the reliability for the visual evoked N170 amplitude in response to happy and neutral faces (0.82 and 0.85) [53].

Interestingly, alpha power showed some differential increases over time. However, as explained in Subjects and Methods, we did not correct for multiple testing. Thus, we are aware that conclusions should be drawn with caution. Within a session, there was an increase in happy-related alpha power for all electrode sites. In contrast, an increase in sad-related alpha power was only seen at the right medioparietal site. This may indicate differences in engagement and/or arousal between both stimuli during the ses- sion [54] and a distinct involvement of parietal cortex areas in emotional processing or related arousal. We also observed alpha power changes across sessions. Alpha power increased at all parietal sites independent of stimulus condition. This may be related to the familiarity of the study procedure at the retest session, evoking less arousal. In contrast, frontal alpha power did not increase at all but only at the frontomedial electrode sites, indicating that the frontal lobe might continuously stay engaged or did not deactivate compared to parietal sites during the processing of affective stimuli even in more familiar low-arousal situations. In an exploratory analysis we investigated the relation between reaction time (time until subjects pressed the button to indicate they detected a change in emotions) and alpha power and asymmetry. Interestingly, alpha power was related to reaction time only in the sad face condition and only when participants were familiar with the task, i.e., in the second block and in the second session: less arousal, indicated by higher alpha power, was related to slower reaction time for sad face detection. For the frontolateral alpha asymmetry, we found a negative correlation between reaction time and sad face detection for the second block and session. This may indicate that a pattern of more right compared to left frontal alpha power - which can be interpreted as approach motivation [25] - was related to shorter reaction time for the detection of the sad face stimulus after a series of happy face stimuli. The significance of these findings will need to be evaluated in studies with larger sample sizes.

There are some important limitations to our study. Firstly, we investigated reliability only in a small sample of healthy individuals. Reliability may also differ in clinical samples. Furthermore, we asked the subjects to rate in how far they were able to empathize with the presented stimuli and to indicate their mood state during the test sessions, but we did not have individual ratings about arousal and valence of the stimuli used. We did not use corrections for multiple testing as explained in the methods section. Lastly, it should be kept in mind that due to absence of studies on stimulus-related alpha asymmetry at present, it is only possible to compare our findings to those studies investigating resting-state data.

\section{Conclusion}

Stimulus-related frontal alpha asymmetry during affective face processing may serve as a useful electrophysiological tool given its moderate to high within-session reliability. However, long-term stability was compara- 
tively low and varied depending on electrode position. Our results also indicate that individual state factors such as affective state or stimulus engagement may influence long-term reliability and should be considered in those studies that investigate treatment effects or the course of illness using alpha asymmetry as an indicator. Our results also provide evidence that frontal alpha asymmetry at medial and lateral sites may represent different physiological processes and thus should be assessed and reported from both positions.

\section{Statement of Ethics}

The study protocol was approved by the institute's committee on human research (Ethics Committee of the Charité - Universitätsmedizin Berlin, Campus Mitte) and was performed in accordance with the ethical standards laid down in the 1964 Declaration of Helsinki. All subjects gave their written informed consent prior to inclusion in the study.

\section{Disclosure Statement}

The authors have no conflicts of interest to declare.

\section{Funding Sources}

J. Rentzsch's work was supported by the Brandenburg Ministry of Science, Research, and Culture.

\section{Author Contributions}

K. Koller-Schlaud and J. Rentzsch supervised the data collection, analyzed the data, and wrote the manuscript. J. Rentzsch designed the experiment. J. Querbach and K. Koller-Schlaud carried out the experiment. J. Behr and A. Ströhle critically reviewed the results and gave substantial input to the manuscript. All authors gave their final approval of the version of the manuscript to be published.

\section{References}

1 Bourke C, Douglas K, Porter R. Processing of facial emotion expression in major depression: a review. Aust N Z J Psychiatry. 2010 Aug;44(8):681-96.

2 Elliott R, Zahn R, Deakin JF, Anderson IM. Affective cognition and its disruption in mood disorders. Neuropsychopharmacology. 2011 Jan;36(1):153-82.

3 Jáni M, Kašpárek T. Emotion recognition and theory of mind in schizophrenia: a meta-analysis of neuroimaging studies. World J Biol Psychiatry. 2018;19(suppl 3):S86-96.

4 Bordon N, O’Rourke S, Hutton P. The feasibility and clinical benefits of improving facial affect recognition impairments in schizophrenia: systematic review and meta-analysis. Schizophr Res. 2017 Oct; 188:3-12.

5 Naranjo C, Kornreich C, Campanella S, Noël X, Vandriette Y, Gillain B, et al. Major depression is associated with impaired processing of emotion in music as well as in facial and vocal stimuli. J Affect Disord. 2011 Feb;128(3):24351.

6 Persad SM, Polivy J. Differences between depressed and nondepressed individuals in the recognition of and response to facial emotional cues. J Abnorm Psychol. 1993 Aug; 102(3):358-68.

7 Rubinow DR, Post RM. Impaired recognition of affect in facial expression in depressed patients. Biol Psychiatry. 1992 May;31(9):947-53.

8 Gopin CB, Burdick KE, Derosse P, Goldberg TE, Malhotra AK. Emotional modulation of response inhibition in stable patients with bipolar I disorder: a comparison with healthy and schizophrenia subjects. Bipolar Disord. 2011 Mar;13(2):164-72.
9 Bermpohl F, Dalanay U, Kahnt T, Sajonz B, Heimann $\mathrm{H}$, Ricken $\mathrm{R}$, et al. A preliminary study of increased amygdala activation to positive affective stimuli in mania. Bipolar Disord. 2009 Feb;11(1):70-5.

10 Stuhrmann A, Suslow T, Dannlowski U. Facial emotion processing in major depression: a systematic review of neuroimaging findings. Biol Mood Anxiety Disord. 2011 Nov; 1(1):10.

11 Bruder GE, Stewart JW, McGrath PJ. Right brain, left brain in depressive disorders: clinical and theoretical implications of behavioral, electrophysiological and neuroimaging findings. Neurosci Biobehav Rev. 2017 Jul;78:178-91.

12 Cantisani A, Koenig T, Horn H, Müller T, Strik W, Walther S. Psychomotor retardation is linked to frontal alpha asymmetry in major depression. J Affect Disord. 2015 Dec;188: $167-72$.

13 Gordon E, Palmer DM, Cooper N. EEG alpha asymmetry in schizophrenia, depression, PTSD, panic disorder, ADHD and conduct disorder. Clin EEG Neurosci. 2010 Oct;41(4): 178-83.

14 Thibodeau R, Jorgensen RS, Kim S. Depression, anxiety, and resting frontal EEG asymmetry: a meta-analytic review. J Abnorm Psychol. 2006 Nov;115(4):715-29.

15 Schaffer CE, Davidson RJ, Saron C. Frontal and parietal electroencephalogram asymmetry in depressed and nondepressed subjects. Biol Psychiatry. 1983 Jul;18(7):753-62.

16 Nusslock R, Walden K, Harmon-Jones E. Asymmetrical frontal cortical activity associated with differential risk for mood and anxiety disorder symptoms: an RDoC perspective. Int $\mathrm{J}$ Psychophysiol. 2015 Nov;98(2 Pt 2):249-61.
17 Kaiser AK, Gnjezda MT, Knasmüller S, Aichhorn W. Electroencephalogram alpha asymmetry in patients with depressive disorders: current perspectives. Neuropsychiatr Dis Treat. 2018 Jun;14:1493-504.

18 Keune PM, Wiedemann E, Schneidt A, Schönenberg M. Frontal brain asymmetry in adult attention-deficit/hyperactivity disorder (ADHD): extending the motivational dysfunction hypothesis. Clin Neurophysiol. 2015 Apr;126(4):711-20.

19 Jaworska N, Blier P, Fusee W, Knott V. Alpha power, alpha asymmetry and anterior cingulate cortex activity in depressed males and females. J Psychiatr Res. 2012 Nov;46(11):1483-91.

20 Kemp AH, Griffiths K, Felmingham KL, Shankman SA, Drinkenburg W, Arns M, et al. Disorder specificity despite comorbidity: resting EEG alpha asymmetry in major depressive disorder and post-traumatic stress disorder. Biol Psychol. 2010 Oct;85(2):3504.

21 Coan JA, Allen JJ, McKnight PE. A capability model of individual differences in frontal EEG asymmetry. Biol Psychol. 2006 May; 72(2):198-207.

22 Debener S, Beauducel A, Nessler D, Brocke B, Heilemann $\mathrm{H}$, Kayser J. Is resting anterior EEG alpha asymmetry a trait marker for depression? Findings for healthy adults and clinically depressed patients. Neuropsychobiology. 2000;41(1):31-7.

23 van der Vinne N, Vollebregt MA, van Putten MJ, Arns M. Frontal alpha asymmetry as a diagnostic marker in depression: fact or fiction? A meta-analysis. Neuroimage Clin. 2017 Jul; 16(July):79-87. 
24 Stewart JL, Coan JA, Towers DN, Allen JJ. Resting and task-elicited prefrontal EEG alpha asymmetry in depression: support for the capability model. Psychophysiology. 2014 May;51(5):446-55.

25 Harmon-Jones E, Gable PA. On the role of asymmetric frontal cortical activity in approach and withdrawal motivation: an updated review of the evidence. Psychophysiology. 2018 Jan;55(1):e12879.

26 Lewis RS, Weekes NY, Wang TH. The effect of a naturalistic stressor on frontal EEG asymmetry, stress, and health. Biol Psychol. 2007 Jul;75(3):239-47.

27 Schmidt B, Hanslmayr S. Resting frontal EEG alpha-asymmetry predicts the evaluation of affective musical stimuli. Neurosci Lett. 2009 Sep;460(3):237-40.

28 Sutton SK, Davidson RJ. Prefrontal brain electrical asymmetry predicts the evaluation of affective stimuli. Neuropsychologia. 2000; 38(13):1723-33.

29 Cheung MC, Law D, Yip J. Evaluating aesthetic experience through personal-appearance styles: a behavioral and electrophysiological study. PLoS One. 2014 Dec;9(12):e115112.

30 Shankman SA, Sarapas C, Klein DN. The effect of pre- vs. post-reward attainment on EEG asymmetry in melancholic depression. Int J Psychophysiol. 2011 Feb;79(2):287-95.

31 Gable P, Harmon-Jones E. Relative left frontal activation to appetitive stimuli: considering the role of individual differences. Psychophysiology. 2008 Mar;45(2):275-8.

32 Schmidt LA, Trainor LJ. Frontal brain electrical activity (EEG) distinguishes valence and intensity of musical emotions. Cogn Emotion. 2001;15(4):487-500.

33 Tenke CE, Kayser J, Pechtel P, Webb CA, Dillon DG, Goer F, et al. Demonstrating test-retest reliability of electrophysiological measures for healthy adults in a multisite study of biomarkers of antidepressant treatment response. Psychophysiology. 2017 Jan;54(1): $34-50$.

34 Tomarken AJ, Davidson RJ, Wheeler RE, Kinney L. Psychometric properties of resting anterior EEG asymmetry: temporal stability and internal consistency. Psychophysiology. 1992 Sep;29(5):576-92.
35 Hagemann D, Naumann E, Thayer JF, Bartussek D. Does resting electroencephalograph asymmetry reflect a trait? an application of latent state-trait theory. J Pers Soc Psychol. 2002 Apr;82(4):619-41.

36 Anokhin AP, Heath AC, Myers E. Genetic and environmental influences on frontal EEG asymmetry: a twin study. Biol Psychol. 2006 Mar;71(3):289-95.

37 Wheeler RE, Davidson RJ, Tomarken AJ. Frontal brain asymmetry and emotional reactivity: a biological substrate of affective style. Psychophysiology. 1993 Jan;30(1):82-9.

38 Rive MM, Mocking RJ, Koeter MW, van Wingen G, de Wit SJ, van den Heuvel OA, et al. State-dependent differences in emotion regulation between unmedicated bipolar disorder and major depressive disorder. JAMA Psychiatry. $2015 \mathrm{Jul} ; 72(7): 687-96$.

39 Sheehan DV, Lecrubier Y, Sheehan KH, Amorim P, Janavs J, Weiller E, et al. The Mini-International Neuropsychiatric Interview (M.I.N.I.): the development and validation of a structured diagnostic psychiatric interview for DSM-IV and ICD-10. J Clin Psychiatry. 1998;59 Suppl 20:22-33.

40 Goeleven E, De Raedt R, Leyman L, Verschuere B. The Karolinska Directed Emotional Faces: A validation study. Cogn Emotion. 2008 Sep;22(6):1094-118.

41 Delorme A, Makeig S. EEGLAB: an open source toolbox for analysis of single-trial EEG dynamics including independent component analysis. J Neurosci Methods. 2004 Mar; 134(1):9-21.

42 Mullen T, Kothe C, Chi YM, Ojeda A, Kerth $\mathrm{T}$, Makeig S, et al. Real-time modeling and 3D visualization of source dynamics and connectivity using wearable EEG. Conf Proc IEEE Eng Med Biol Soc. 2013;2013:2184-7.

43 Gómez-Herrero G, De Clercq W, Anwar H, Kara O, Egiazarian K, Van Huffel S, et al. Automatic removal of ocular artifacts in the EEG without an EOG reference channel. Proceedings of the 7th Nordic Signal Processing Symposium - NORSIG 2006, Reykjavik, 2006, p. 130-3.

44 Rentzsch J, Jockers-Scherübl MC, Boutros NN, Gallinat J. Test-retest reliability of P50, N100 and P200 auditory sensory gating in healthy subjects. Int J Psychophysiol. 2008 Feb;67(2):81-90.
45 Jesulola E, Sharpley CF, Bitsika V, Agnew LL, Wilson P. Frontal alpha asymmetry as a pathway to behavioural withdrawal in depression: research findings and issues. Behav Brain Res. 2015 Oct;292:56-67.

46 Winegust AK, Mathewson KJ, Schmidt LA. Test-retest reliability of frontal alpha electroencephalogram (EEG) and electrocardiogram (ECG) measures in adolescents: a pilot study. Int J Neurosci. 2014 Dec;124(12):908-11.

47 Harmon-Jones E, Lueck L, Fearn M, Harmon-Jones C. The effect of personal relevance and approach-related action expectation on relative left frontal cortical activity. Psychol Sci. 2006 May; 17(5):434-40.

48 Nusslock R, Shackman AJ, Harmon-Jones E, Alloy LB, Coan JA, Abramson LY. Cognitive vulnerability and frontal brain asymmetry: common predictors of first prospective depressive episode. J Abnorm Psychol. 2011 May;120(2):497-503.

49 Choi D, Sekiya T, Minote N, Watanuki S. Relative left frontal activity in reappraisal and suppression of negative emotion: evidence from frontal alpha asymmetry (FAA). Int J Psychophysiol. 2016 Nov; 109:37-44.

50 Quaedflieg CW, Meyer T, Smulders FT, Smeets T. The functional role of individualalpha based frontal asymmetry in stress responding. Biol Psychol. 2015 Jan;104:75-81.

51 Prause N, Staley C, Roberts V. Frontal alpha asymmetry and sexually motivated states. Psychophysiology. 2014 Mar;51(3):226-35.

52 Stewart JL, Coan JA, Towers DN, Allen JJ. Frontal EEG asymmetry during emotional challenge differentiates individuals with and without lifetime major depressive disorder. J Affect Disord. 2011 Mar;129(1-3):167-74.

53 Cassidy SM, Robertson IH, O'Connell RG. Retest reliability of event-related potentials: evidence from a variety of paradigms. Psychophysiology. 2012 May;49(5):659-64.

54 Speliotes EK, Willer CJ, Berndt SI, Monda KL, Thorleifsson G, Jackson AU, et al.; MAGIC; Procardis Consortium. Association analyses of 249,796 individuals reveal 18 new loci associated with body mass index. Nat Genet. 2010 Nov;42(11):937-48. 